Send your letters to the Editor,

British Dental Journal,

64 Wimpole Street

London

W1G 8YS

Email bdj@bda.org

Priority will be given to letters less than 500 words long.

Authors must sign the letter, which

may be edited for reasons of space.

\section{CHECK THE FACTS}

Sir, on the letters page of the 14 August $B D J$, D. Burton finishes their letter Diagnosis tosh with the words 'Something's wrong somewhere!' Might I suggest that what is wrong is taking the word of a patient without hearing the dentist's side of the story? As every dentist in general practice knows, sometimes patients don't listen and occasionally they have even been known to tell a fib. I am unsure if the intention of writing the letter was, by implication, to denigrate two dentists apparently working under NHS contract, or to highlight the problems of the contract itself. Either way, and I suspect the former, it might have been wise to check the facts with the dentists concerned first.

R. Elvin

Watford

DOI: 10.1038/sj.bdj.2010.929

\section{DESPERATELY PATRONISING}

Sir, firstly congratulations on an excellent editorial (BDJ 2010; 209: 103); your restraint and moderation is admirable in the circumstances.

The thrust of this unbelievable resurrection of the amazingly silly issue of the use of the title 'Dr' is found in the paragraph within the draft GDC consultation document (destined to never be read - let alone commented upon - by the vast majority of registrants) that reads ‘...its use as a courtesy title (ie Doctor) is potentially misleading to patients and that it is important that patients do not assume that you have training and competencies which you do not possess' (italics and bold are mine).

What desperately patronising and insulting tosh. Firstly, what pray are 'these standards and competencies' that are appropriate and essential to justify the use of the title 'Dr' by anyone, and if not those of that excellent healthcare professional called a dentist - then who and what? As a holder of a higher doctorate, and not quite open therefore to being judged as having an 'axe to grind', I believe the title of doctor is the only appropriate title for all dentists. Are the GDC still stuck in the era when they thought that dentists called 'Dr' were trying to impersonate the GMP or other similar healthcare professional? If so, why on earth would they want to do such?

Currently dentists are trained in parallel with, and alongside, medics in human disease and the medical skills of surgery, prescribing, injecting drugs, the spectrum of diagnosis and generally dealing with, and accepting responsibility for, patients on a whole person basis and all that this involves. These skills will be needed often against a background of polypharmacy, varying degrees of wellness and illness for patients treated, comprising the very young to the geriatric and frail. Mistakes are known to have a significant effect! Those that see this title nonsense as a problem within the GDC should visit ActE departments, the Armed Forces, teaching hospitals and/or watch an expert implant team at work and they will find the status, training and competencies of the dentist are certainly not in doubt and more than equal to any of the other healthcare professionals that they work with and alongside in some of the aforementioned teams.

Why is it that the mouth and oral disease are considered some totally different deal as if outside the body by this hopeless GDC? Is dentistry rather than being a specialised branch of medicine and healthcare seen as some sort of isolated procedure akin to jobbing carpentry?
An obvious and final solution should be that training programmes should be brought back into the medical umbrella and ALL medical specialities (dentistry would be one with its own sub-specialities) then begin with a three or four year generalist training prior to further more advanced and specific programmes for eventual specialists and/or advanced generalists.

In this way, dentistry would return to the fold of the General Medical Council (GMC) and the cash-greedy, outdated, poor value and patronising GDC could be abolished.

Bring it on!

K. Marshall

By email

DOI: 10.1038/sj.bdj.2010.930

\section{NIRVANA IMPEDIMENT}

Sir, in the article Rethinking ferrule - a new approach to an old dilemma (BDJ 2010; 209: 25-33) the authors advocate the adoption of a classification system based upon the 'pooled' 'available' 'evidence' base relating to the ferrule effect and a related algorithm to assist clinicians. However, they acknowledge that it was deficient in a number of areas and furthermore, despite their efforts, clinicians using the system are left with a large number of factors to consider and could not reasonably be expected to reliably choose the optimum combined biomechanical and aesthetic outcome.

The authors do cite a small number of references relating to computational stress analysis (finite element analysis [FEA]), though a PubMed search for 'finite element analysis ferrule' returned eight papers, seven of which were published in 2009 or before, yet only two of which were not cited in the article. 
FEA is a technology which will probably be a pivotal element of the eventual solution not only to the ferrule issue but tooth preparation prior to final restoration in general, through its incorporation into commercial high resolution dental optical impression - CAD/CAM (computer aided design, computer aided manufacture) systems - very probably with additional modules for defining:

- The remaining tooth structure following decay and/or previous restoration debridement

- Areas where adhesion would not be achieved (eg dental linings)

- The required aesthetic outcome (most critically minimum thickness of aesthetic material - restorative or tooth substance)

- Occlusal force assessment derived from an analysis of the optical registration for wear pattern.

When combined with intelligent agents for generating minimum cavity preparation and optimum restorative system (material/s and adhesive/s) solutions, then clinicians will have a comprehensive evidence-supported answer to the issue.

Recently, reassurance in respect of viability of FEA in this application was provided by Schmitter et al. ${ }^{1}$ who conducted in vitro and corresponding computational studies on fibre post supported restorations, variables in which included ferrule height and cementation method. The authors reported that 'FEA confirmed these [in vitro] results and provided information about stress and force distribution within the restoration'.

With dentine bonding systems now achieving shear strength values approximately one third ${ }^{2}$ of the average for dentine itself, ${ }^{3}$ there would appear to be an open door to predictable, consistent, optimised and evidence-based restorative care at the single tooth level.

The only real potential impediment to such a nirvana are the commercial models which holders of optical impression software intellectual property rights will adopt in order to promote own brand and partner dental materials.

$$
\begin{array}{r}
\text { P. Mc Crory } \\
\text { By email }
\end{array}
$$

1. Schmitter M, Rammelsberg P, Lenz J et al. Teeth restored using fiber-reinforced posts: in vitro fracture tests and finite element analysis. Acta Biomater 2010; 6: 3747-3754.

2. Graiff L, Piovan C, Vigolo P, Mason P N. Shear bond strength between feldspathic CAD/CAM ceramic and human dentine for two adhesive cements. J Prosthodont 2008; 17: 294-299.

3. Watanabe L G, Marshall G W Jr, Marshall S J. Dentin shear strength: effects of tubule orientation and intratooth location. Dent Mater 1996; 12: 109-115.

DOI: 10.1038/sj.bdj.2010.931

\section{HERBAL HISTORY}

Sir, I read with great interest the letter to the editor entitled Herbal interactions (BDJ 2010; 209: 57). There has been an increase in the use of herbal medicines around the world in recent years in the general belief amongst the public that they are safe because they are obtained from natural sources. However, some of these products have associated adverse effects. Ginger contains gingerols and shogaols that prevent blood cells from clotting and acts as a natural blood thinner. Similarly, garlic is extremely beneficial to the entire cardiovascular system. Chemicals named alliin, allinase and allicin present in garlic improve blood circulation by thinning the blood.

Ginkgo and ginseng herbal supplements are widely used in Europe and the United States to treat circulatory disorders. Ginkgo leaves contain two types of chemicals (flavonoids and terpenoids). Flavonoids protect the blood vessels from damage and terpenoids improve blood flow by dilating blood vessels and reducing the stickiness of platelets. Though all these herbal medicines have a beneficial blood thinning action especially in heart diseases, they can be risky to consume in large quantities. The blood thinning effect of these natural products may lead to bleeding from the gums, nose, in urine, stools and bruises. Hence, patients should never start self medication and combine artificial and natural blood thinners without consulting a physician. These herbal supplements should be stopped by patients at least 36 hours prior to surgery or dental procedures due to the risk of bleeding complications. The medical history taken by the dentist should include questions regarding the taking of herbal and over-the-counter medications.

\section{A. Parolia, Karnataka} By email 